\title{
Cycles de codimension 2 en produits de variétés de Severi-Brauer
}




\title{
CYCLES DE CODIMENSION 2 EN PRODUITS DE VARIÉTÉS DE SEVERI-BRAUER
}

\author{
NIKITA A. KARPENKO
}

RÉSUMÉ. Nous recherchons le groupe de Chow des cycles de codimension 2 en le produit de $n$ variétés de Severi-Brauer $(n \geq 2)$. Nous analysons plus en détail

- le produit d'une variété biquaternionique et d'une conique;

- le produit de deux surfaces de Severi-Brauer.

\section{TABLE DES MATIÈRES}

0. Introduction

1. Deux résultats préliminaires

2. Groupe de Grothendieck de produit de variétés de Severi-Brauer

3. Variétés et algèbres disjointes

4. Variétés "génériques"

5. Algèbres "génériques"

6. Variété biquaternionique fois conique

7. Produit de deux surfaces de Severi-Brauer

Références 


\section{INTRODUCTION}

Dans [9], nous recherchions le groupe de Chow $\mathrm{CH}^{2}$ pour une variété de Severi-Brauer. Ici, en utilisant les mêmes méthodes, nous recherchons le même groupe pour un produit direct de mêmes variétés. La motivation pour ce travail est donnée par un résultat de Izhboldin ([5]): le corps de fonctions d'une variété de Severi-Brauer est universellement excellent ssi l'indice de l'algèbre correspondante n'est pas divisible par 4. Cependant, pour ce résultat, il avait besoin d'information certaine sur le groupe $\mathrm{CH}^{2}$ du produit d'une variété biquaternionique et d'une conique (6.1).

Des premiers résultats sur le groupe $\mathrm{CH}^{2}$ d'un produit de variétés de SeveriBrauer sont obtenus dans [14]. On y établit sa connexion avec le 3-ième groupe de cohomologie galoisienne ([14, theorem 4.1]) et on y construit un exemple d'un produit de trois coniques avec torsion dans $\mathrm{CH}^{2}$ ([14, remark 6.1]).

Le résultat principal et général de l'article présent est 5.5 (avec le corollaire 5.6). Nous l'appliquons à des produits de deux variétés de petites dimensions (6.1), (7.1); on arrive en particulier à des exemples nouveaux de torsion dans $\mathrm{CH}^{2}$.

Nous utilisons la terminologie et la notation suivantes. Si on $\operatorname{dit}$ que $A$ est une algèbre, on veut toujours dire que $A$ est une algèbre centrale simple sur un corps. Pour une algèbre $A$ sur un corps $F$ on note $[A]$ sa classe dans le groupe de $\operatorname{Brauer} \operatorname{Br}(F)$ de $F$; exp $A$ désigne l'exposant, deg $A$ le degré et ind $A$ l'indice de $A$.

La variété de Severi-Brauer d'une algèbre $A$ est désignée par $\mathrm{SB}(A)$. Une variété est toujours une variété algébrique projective lisse sur un corps; un faisceau sur $X$ est un $\mathcal{O}_{X}$-module. L'anneau de Grothendieck d'une variété $X$ est désigné par $K(X)$;

$$
K(X)=\Gamma^{0} K(X) \supset \Gamma^{1} K(X) \supset \ldots \text { et } K(X)=\mathrm{T}^{0} K(X) \supset \mathrm{T}^{1} K(X) \supset \ldots
$$

sont respectivement la gamma-filtration et la filtration topologique de $K(X)$; on utilise la notation $\mathrm{G}^{*} \Gamma K(X)$ et $\mathrm{G}^{*} \mathrm{~T} K(X)$ pour les anneaux adjoints gradués de ces filtrations. Il y a des relations certaines entre $\mathrm{G}^{*} \Gamma K(X), \mathrm{G}^{*} \mathrm{~T} K(X)$ et l'anneau de Chow $\mathrm{CH}^{*}(X)$ que nous utilisons mais ne décrivons pas ici; on les trouve dans $[9, \S 2]$.

Ce travail est exécuté au Labo de Mathématiques de l'Université de FrancheComté. Je le remerci sincèrement pour l'atmosphère et les conditions excellentes.

\section{DeUX RÉSUltats PRÉliminaires}

Proposition 1.1. Soient $A_{1}, \ldots, A_{n}$ et $B_{1}, \ldots, B_{m}$ des algèbres sur un corps $F$ telles que les sous-groupes de $\operatorname{Br}(F)$ engendrés par $\left[A_{1}\right], \ldots,\left[A_{n}\right]$ et par $\left[B_{1}\right], \ldots,\left[B_{m}\right]$ coincident. Alors

$$
\text { Tors } \mathrm{CH}^{2}\left(\mathrm{SB}\left(A_{1}\right) \times \cdots \times \mathrm{SB}\left(A_{n}\right)\right) \simeq \operatorname{Tors~} \mathrm{CH}^{2}\left(\mathrm{SB}\left(B_{1}\right) \times \cdots \times \mathrm{SB}\left(B_{m}\right)\right) \text {. }
$$


Démonstration. Posons $X=\mathrm{SB}\left(A_{1}\right) \times \cdots \times \mathrm{SB}\left(A_{n}\right), Y_{1}=\mathrm{SB}\left(B_{1}\right)$. Il suffit de montrer que

$$
\text { Tors } \mathrm{CH}^{2}(X) \simeq \text { Tors } \mathrm{CH}^{2}\left(X \times Y_{1}\right) \text {. }
$$

Puisque $X \times Y_{1} \rightarrow X$ est une fibration projective (5.3), on a ([4, $\S 2$ of appendix A])

$$
\mathrm{CH}^{2}\left(X \times Y_{1}\right) \simeq \mathrm{CH}^{2}(X) \oplus \cdots \oplus \mathrm{CH}^{2-\operatorname{dim} Y_{1}}(X) \text {. }
$$

La dernière observation est: pour tout $i<2$, le groupe $\mathrm{CH}^{i}(X)$ n'a pas de torsion (pour $i=1 \mathrm{v}$. $[16$, lemme 6.3, (i)]).

Soit $p$ un premier. Pour une algèbre ainsi que pour un groupe abélien $A$, on va noter $A\{p\}$ la partie $p$-primaire de $A$.

Proposition 1.2. Soient $A_{1}, \ldots, A_{n}$ des algèbres sur un (même) corps. On a $\mathrm{CH}^{2}\left(\mathrm{SB}\left(A_{1}\right) \times \cdots \times \mathrm{SB}\left(A_{n}\right)\right)\{p\} \simeq \operatorname{Tors} \mathrm{CH}^{2}\left(\mathrm{SB}\left(A_{1}\{p\}\right) \times \cdots \times \mathrm{SB}\left(A_{n}\{p\}\right)\right)$.

Démonstration. Pour $n=1$, le résultat est démontré dans [9, proposition 1.3]. La même preuve marche pour $n>1$.

\section{Groupe de GRothendiECK DE PRODUIT DE VARIÉtÉS DE SEVERI-BRAUER}

Soient $A_{1}, \ldots, A_{n}$ des algèbres sur un corps $F, X_{1}, \ldots, X_{n}$ leurs variétés de Severi-Brauer et $X=X_{1} \times \cdots \times X_{n}$. Fixons une clôture séparable $\bar{F}$ de $F$ et posons $\bar{X}_{i}=\left(X_{i}\right)_{F}$ pour chaque $i$. Les variétés $\bar{X}_{i}$ sont des espaces projectifs; notons $\xi_{i}$ la classe dans $K(\bar{X})$ du faisceau tautologique de la fibration projective

$$
\bar{X} \rightarrow \prod_{j \neq i} \bar{X}_{j} .
$$

L'anneau $K(\bar{X})$ est engendré par les éléments $\xi_{1}, \ldots, \xi_{n}$ avec les seules relations

$$
\left(\xi_{1}-1\right)^{\operatorname{deg} A_{1}}=\cdots=\left(\xi_{n}-1\right)^{\operatorname{deg} A_{n}}=0 \text {. }
$$

Regardons la restriction $K(X) \rightarrow K(\bar{X})$ qui est un homomorphisme d'anneaux.

Théorème 2.1. L'homomorphisme $K(X) \rightarrow K(\bar{X})$ est injectif; son image est additivement engendrée par les éléments

$$
\operatorname{ind}\left(A_{1}^{\otimes j_{1}} \otimes \cdots \otimes A_{n}^{\otimes j_{n}}\right) \cdot \xi_{1}^{j_{1}} \cdots \xi_{n}^{j_{n}}
$$

avec $0 \leq j_{1}<\operatorname{deg} A_{1}, \ldots, 0 \leq j_{n}<\operatorname{deg} A_{n}$.

Démonstration. On utilise $[15, \S 8$, theorem 4.1] $n$ fois.

Corollaire 2.2. Pour des algèbres $A_{1}, \ldots, A_{n}$ des degrés fixés, l'anneau $K(X)$ avec la gamma-filtration (et le groupe Tors $\mathrm{G}^{2} \Gamma K(X)$ en particulier) dépend seulement des nombres ind $\left(A_{1}^{\otimes j_{1}} \otimes \cdots \otimes A_{n}^{\otimes j_{n}}\right)$.

Démonstration. Par le théorème, les nombres déterminent $K(X)$ entièrement comme un sous-anneau de $K(\bar{X})$. Les classes de Chern à valeurs dans $K([9$, definition 2.1]) pour $X$ qui déterminent la gamma-filtration ([9, definition 2.6]) sont les restrictions des classes de Chern pour $\bar{X}$. 


\section{VARIÉTÉS ET ALGÈBRES DISJOINTES}

Définition 3.1. Soient $X_{1}, \ldots, X_{n}$ des variétés quelconques sur un (même) corps. Nous disons qu'elles sont disjointes si l'homomorphisme d'anneaux

$$
K\left(X_{1}\right) \otimes \cdots \otimes K\left(X_{n}\right) \rightarrow K\left(X_{1} \times \cdots \times X_{n}\right),
$$

induit par les homomorphismes d'image inverse

$$
p r_{i}^{*}: K\left(X_{i}\right) \rightarrow K\left(X_{1} \times \cdots \times X_{n}\right)
$$

par rapport aux projections $p r_{i}: X_{1} \times \cdots \times X_{n} \rightarrow X_{i}$, est un isomorphisme.

Proposition 3.2. Soient $X_{1}, \ldots, X_{n}$ des variétés disjointes. La gamma-filtration de $K\left(X_{1} \times \cdots \times X_{n}\right)$ coincide avec la filtration induite par les gammafitrations de $K\left(X_{1}\right), \ldots, K\left(X_{n}\right)$.

Démonstration. Notons $X$ le produit $X_{1} \times \cdots \times X_{n}$ et $\tilde{\Gamma}$ la filtration induite. Alors, pour chaque $l \geq 0$, on définit $\tilde{\Gamma}^{l} K(X)$ comme le sous-groupe de $K(X)$ engendré par tous les produits

$$
p r_{1}^{*} \Gamma^{l_{1}} K\left(X_{1}\right) \cdots p r_{n}^{*} \Gamma^{l_{n}} K\left(X_{n}\right)
$$

avec $l_{1}+\cdots+l_{n} \geq l$. Comme un homomorphisme d'image inverse respecte la gamma-filtration, on a l'inclusion $\tilde{\Gamma}^{l} K(X) \subset \Gamma^{l} K(X)$. Démontrons l'inclusion inverse. Puisque la gamma-filtration $\Gamma$ de $K(X)$ est la plus petite filtration d'anneau ayant les propriétés $\Gamma^{0} K(X)=K(X)$ et $c^{l}(x) \in \Gamma^{l} K(X)$ pour tout $x \in K(X)$ et $l \geq 1$, où $c^{l}$ est la $l$-ième classe de Chern à valeurs dans $K([9$, definition 2.1]), il suffit de montrer que

$$
c^{l}(x) \in \tilde{\Gamma}^{l} K(X) .
$$

Comme les variétés $X_{1}, \ldots, X_{n}$ sont disjointes, le groupe additif de $K(X)$ est engendré par les produits

$$
x=p r_{1}^{*}\left(x_{1}\right) \cdots p r_{n}^{*}\left(x_{n}\right)
$$

où $x_{i} \in K\left(X_{i}\right)$ est la classe d'un faisceau localement libre. Il suffit donc de vérifier l'inclusion (*) seulement pour $x$ de la forme (**). Puisque $c^{l}$ commute avec $p r_{i}^{*}$, on a

$$
c^{l}\left(p r_{i}^{*}\left(x_{i}\right)\right) \in \tilde{\Gamma}^{l} K(X),
$$

et le dernier pas de la preuve est

Lemme 3.3. Soient $n, m, l \geq 0$. Il existe un polynôme $f_{l}\left(\left(\sigma_{i}\right),\left(\tau_{j}\right)\right)$ sur $\mathbb{Z}$ en les variables $\sigma_{1}, \ldots, \sigma_{n}$ et $\tau_{1}, \ldots, \tau_{m}$ ayant les deux propriétés suivantes:

- si $x, y \in K(X)$ sont les classes de faisceaux localement libres sur une variété $X$, la classe de Chern $c^{l}(x \cdot y)$ est égale à $f_{l}\left(c^{i}(x), c^{j}(y)\right)$;

- si on pose $\operatorname{deg} \sigma_{i}=i$ et $\operatorname{deg} \tau_{j}=j$, le degré de chaque monôme de $f_{l}$ est supérieur ou égal à l. 
Démonstration. Par le principe de décomposition ([13, prop. 5.6]), il suffit de regarder le cas où

$$
x=\xi_{1}+\cdots+\xi_{n}, \quad y=\eta_{1}+\cdots+\eta_{m}
$$

avec les classes de faisceaux inversibles $\xi_{i}, \eta_{j}$. Pour la classe de Chern totale $c_{t}$ ([9, definition 2.1]), on a

$$
\begin{aligned}
c_{t}(x) & =c_{t}\left(\sum_{i=1}^{n} \xi_{i}\right)=\prod_{i=1}^{n}\left(1+\left(\xi_{i}-1\right) t\right)=\prod_{i=1}^{n}\left(1+a_{i} t\right) \text { où } a_{i}=\xi_{i}-1 \\
c_{t}(y) & =c_{t}\left(\sum_{j=1}^{m} \eta_{j}\right)=\prod_{j=1}^{m}\left(1+\left(\eta_{j}-1\right) t\right)=\prod_{j=1}^{m}\left(1+b_{j} t\right) \text { où } b_{j}=\eta_{j}-1 ; \\
c_{t}(x y) & =c_{t}\left(\sum_{i, j} \xi_{i} \eta_{j}\right)=\prod_{i, j}\left(1+\left(\xi_{i} \eta_{j}-1\right) t\right)=\prod_{i, j}\left(1+\left(a_{i} b_{j}+a_{i}+b_{j}\right) t\right) .
\end{aligned}
$$

La classe $c^{l}(x y)$ est (par définition) le coefficient de $t^{l}$ dans $c_{t}(x y)$. Ce coefficient est évidemment un polynôme en $a_{1}, \ldots, a_{n}$ et $b_{1}, \ldots, b_{m}$ qui est symétrique par rapport aux variables $\left(a_{i}\right)$ et aussi symétrique par rapport aux variables $\left(b_{j}\right)$ (notons que le degré de chaque monôme est au moins égal à $l$ ). Alors, par le théorème fondamental sur les polynômes symétriques, $c^{l}(x y)=f_{l}\left(\left(\sigma_{i}\right),\left(\tau_{j}\right)\right)$ pour un polynôme $f_{l}$, où $\left(\sigma_{i}\right)_{i=1}^{n}$ sont les polynôme symétriques élémentaires en $\left(a_{i}\right)$ ( $\sigma_{i}$ est un polynôme homogène de degré $i$ ) et $\left(\tau_{j}\right)_{j=1}^{m}$ les polynôme symétriques élémentaires en $\left(b_{j}\right)$. L'assertion de la lemme concernant le degré est évidemment satisfaite. Finalement, on note que $\sigma_{i}=c^{i}(x)$ et $\tau_{j}=c^{j}(y)$.

Corollaire 3.4. Soient $X_{1}, \ldots, X_{n}$ des variétés telles que leurs groupes de Grothendieck sont finement engendrés (des variétés de Severi-Brauer par exemple). Si les variétés sont disjointes et les groupes $\mathrm{G}^{*} \Gamma K\left(X_{1}\right), \ldots, \mathrm{G}^{*} \Gamma K\left(X_{n}\right)$ n'ont pas de torsion, le groupe $\mathrm{G}^{*} \Gamma K\left(X_{1} \times \cdots \times X_{n}\right)$ est aussi sans torsion.

Démonstration. L'homomorphisme naturel

$$
\mathrm{G}^{*} \Gamma K\left(X_{1}\right) \otimes \cdots \otimes \mathrm{G}^{*} \Gamma K\left(X_{n}\right) \rightarrow \mathrm{G}^{*} \Gamma K\left(X_{1} \times \cdots \times X_{n}\right)
$$

est surjectif par la proposition. Par notre condition, le groupe du côté gauche est finement engendré et sans torsion; alors c'est un groupe libre abélien de rang fini. Ce rang coïncide avec le rang du groupe du côté droit parce que les variétés sont disjointes.

Maintenant, on va comprendre ce que la condition d'être disjointes veut dire pour des variétés de Severi-Brauer.

Définition 3.5. Soient $A_{1}, \ldots, A_{n}$ des algèbres sur un (même) corps. On dit qu'elles sont disjointes si

$$
\operatorname{ind}\left(A_{1}^{\otimes j_{1}} \otimes \cdots \otimes A_{n}^{\otimes j_{n}}\right)=\operatorname{ind} A_{1}^{\otimes j_{1}} \cdots \text { ind } A_{n}^{\otimes j_{n}} \text { pour tous } j_{1}, \ldots, j_{n} \geq 0 \text {. }
$$

Proposition 3.6. Des algèbres $A_{1}, \ldots, A_{n}$ sont disjointes ssi leurs variétés de Severi-Brauer sont disjointes. 
Démonstration. Comme pour une algèbre $A$ arbitraire il y a un isomorphisme canonique $K(A)=$ ind $A \cdot \mathbb{Z}$ où maintenant $K$ note le groupe de Grothendieck de l'algèbre, les algèbres sont disjointes ssi les applications

$$
K\left(A_{1}^{\otimes j_{1}}\right) \otimes \cdots \otimes K\left(A_{n}^{\otimes j_{n}}\right) \rightarrow K\left(A_{1}^{\otimes j_{1}} \otimes \cdots \otimes A_{n}^{\otimes j_{n}}\right)
$$

sont des isomorphismes pour tous $0 \leq j_{1}<\operatorname{deg} A_{1}, \ldots, 0 \leq j_{n}<\operatorname{deg} A_{n}$. En prenant la somme directe, on obtient l'application

$$
\begin{aligned}
\left(\coprod_{j_{1}=0}^{\operatorname{deg} A_{1}-1} K\left(A_{1}^{\otimes j_{1}}\right)\right) \otimes \cdots \otimes & \left(\coprod_{j_{n}=0}^{\operatorname{deg} A_{n}-1} K\left(A_{n}^{\otimes j_{n}}\right)\right) \longrightarrow \\
& \longrightarrow \coprod_{j_{1}=0}^{\operatorname{deg} A_{1}-1} \cdots \coprod_{j_{n}=0}^{\operatorname{deg} A_{n}-1} K\left(A_{1}^{\otimes j_{1}} \otimes \cdots \otimes A_{n}^{\otimes j_{n}}\right) .
\end{aligned}
$$

En identifiant les facteurs du produit du côté gauche avec $K\left(\operatorname{SB}\left(A_{1}\right)\right), \ldots$, $K\left(\mathrm{SB}\left(A_{n}\right)\right)$ et la somme directe du côté droit avec $K\left(\operatorname{SB}\left(A_{1}\right) \times \cdots \times \mathrm{SB}\left(A_{n}\right)\right)$ par 2.1, on obtient à la place de la flèche l'homomorphisme de 3.1.

\section{VARIÉTÉS "GÉNÉRIQUES"}

Définition 4.1. Disons que une variété $X$ est "générique" si la gamma-filtration de $K(X)$ coïncide avec la filtration topologique.

Lemme 4.2. Si Tors $\mathrm{G}^{*} \Gamma K(X)=0$ (pour une variété arbitraire $X$ ), alors $X$ est "générique".

Démonstration. Pour voir que les filtrations coïncident, il suffit de montrer que l'homomorphisme

$$
\alpha: \mathrm{G}^{*} \Gamma K(X) \rightarrow \mathrm{G}^{*} \mathrm{~T} K(X),
$$

induit par l'inclusion des filtrations, est injectif. Comme $\alpha \otimes \mathbb{Q}$ est bijectif ([3, proposition 5.5 of chapter VI]), le noyau de $\alpha$ contient seulement des éléments d'ordre fini. Donc $\alpha$ est vraiment injectif si le groupe $\mathrm{G}^{*} \Gamma K(X)$ est sans torsion.

Lemme 4.3. Soit $\mathcal{G} \rightarrow X$ une fibration grassmannienne. Si $X$ est "générique", la variété $\mathcal{G}$ est pareillement "générique".

Démonstration. Puisque $\mathcal{G}$ est une fibration grassmannienne sur $X$, la $\mathrm{CH}^{*}(X)$ algèbre (commutative) $\mathrm{CH}^{*}(\mathcal{G})$ est engendrée par les classes de Chern (à valeurs dans $\left.\mathrm{CH}^{*}\right)$ (v. [2, proposition 14.6.5] ou [11, (3.2)]). En utilisant l'épimorphisme naturel $\mathrm{CH}^{*} \rightarrow \mathrm{G}^{*} \mathrm{~T} K$, on obtient le même résultat pour $\mathrm{G}^{*} \mathrm{~T} K:$ la $\mathrm{G}^{*} \mathrm{~T} K(X)$ algèbre (commutative) $\mathrm{G}^{*} \mathrm{~T} K(\mathcal{G})$ est engendrée par les classes de Chern (à valeurs dans $\left.\mathrm{G}^{*} \mathrm{~T} K\right)$. Comme $X$ est "générique", l'anneau $\mathrm{G}^{*} \mathrm{~T} K(X)$ même est engendré par les classes de Chern ([9, remark 2.17]). Par conséquent, $\mathrm{G}^{*} \mathrm{~T} K(\mathcal{G})$ est engendré par les classes de Chern non seulement comme l'algèbre mais aussi comme un anneau. Cela veut dire que $\mathcal{G}$ est "générique" ([9, remark 2.17]). 
Lemme 4.4. Soit $X \rightarrow Y$ un morphisme lisse de variétés, $\tilde{X}$ sa fibre générique. Si $X$ est "générique", la variété $\tilde{X}$ (c'est une variété sur le corps de fonctions de $Y$ ) est aussi "générique".

Démonstration. Le morphisme (de schémas) $\tilde{X} \rightarrow X$ induit un homomorphisme des groupes de Grothendieck $K(X) \rightarrow K(\tilde{X})$, respectant les deux filtrations, et un homomorphisme des groupes de $\mathrm{Chow} \mathrm{CH}^{*}(X) \rightarrow \mathrm{CH}^{*}(\tilde{X})$ qui est surjectif ([10, theorem 3.1]). Par conséquent, l'homomorphisme

$$
\mathrm{G}^{*} \mathrm{~T} K(X) \rightarrow \mathrm{G}^{*} \mathrm{~T} K(\tilde{X})
$$

est aussi surjectif, et donc, pour chaque $l$, le groupe $\mathrm{T}^{l} K(X)$ est appliqué surjectif dans $\mathrm{T}^{l} K(\tilde{X})$. Puisque $\mathrm{T}^{l} K(X)=\Gamma^{\prime} K(X)$, on en déduit que $\mathrm{T}^{l} K(\tilde{X}) \subset$ $\Gamma^{l} K(\tilde{X})$. L'inclusion inverse a lieu tout le temps.

Corollaire 4.5. Soient $X$ et $Y$ des variétés sur un corps $F$ telles que la projection $X \times Y \rightarrow Y$ est une fibration grassmannienne. Si $X$ est "générique", alors $X_{F(Y)}$ est pareillement "générique".

Démonstration. La variété $X \times Y$ est "générique" d'après 4.3; ensuite la variété $X_{F(Y)}$ est "générique" par 4.4 .

\section{AlgÈbres “GÉNÉRIQUES"}

Proposition 5.1. Soit $A$ une algèbre primaire (i.e. $\operatorname{deg} A$ est une puissance d'un premier). On suppose que

- soit ind $A=\exp A$

- soit ind $A=2^{n}$ et ind $A^{\otimes 2^{n-2}}=4(n \geq 2)$

(une algèbre des biquaternions est un exemple de telle $A$ ). Alors, le groupe $\mathrm{G}^{*} \Gamma(\mathrm{SB}(A))$ n'a pas de torsion.

Démonstration. Pour des algèbres du premier type, v. [9, proposition 3.3, corollary 3.6]; pour le deuxième type, v. la démonstration de [9, proposition 4.9].

Corollaire 5.2. Soient $A_{1}, \ldots, A_{n}$ des algèbres disjointes et supposons que chaque $A_{i}$ satisfait la condition de 5.1. Alors pour le produit $X$ de leurs variétés de Severi-Brauer, on a: Tors $\mathrm{G}^{*} \Gamma K(X)=0$; en particulier, Tors $\mathrm{CH}^{2}(X)=0$.

Démonstration. Ceci résulte directement de la proposition avec 3.4 et 3.6.

Pour une algèbre $B$ et un entier $r \geq 0$, notons $\operatorname{SB}(r, B)$ la variété de SeveriBrauer généralisée des idéaux à droite de $B$ de rang $r([1, \S 2])$. En particulier, $\mathrm{SB}(1, B)=\mathrm{SB}(B)$.

Proposition 5.3. Soient $A_{1}, \ldots, A_{n}$ et $B$ des algèbres sur un (même) corps, $X=\mathrm{SB}\left(A_{1}\right) \times \cdots \times \mathrm{SB}\left(A_{n}\right)$ et $Y=\mathrm{SB}(r, B)$ avec certain $r \geq 0$.

Si la classe $[B]$ de l'algèbre $B$ dans le groupe de Brauer appartient au groupe engendré par $\left[A_{1}\right], \ldots,\left[A_{n}\right]$, la projection $X \times Y \rightarrow X$ est une fibration $r$ grassmannienne. 
Démonstration. On peut supposer que

$$
B \simeq A_{1}^{\otimes j_{1}} \otimes \cdots \otimes A_{n}^{\otimes j_{n}}
$$

avec $j_{1}, \ldots, j_{n} \geq 0$ certains. Considérons le carré cartésien



où $T=\mathrm{SB}(B)$ et le morphisme $X \rightarrow T$ est donné par le produit tensoriel d'idéaux. La flèche du côté droit (c'est la projection $T \times Y \rightarrow T$ ) est une fibration $r$-grassmannienne d'après [9, proposition 6.3]. Donc, la projection $X \times Y \rightarrow Y$ (c'est la flèche du côté gauche) est aussi une fibration $r$-grassmannienne.

Définition 5.4. Disons que une collection d'algèbres $\tilde{A}_{1}, \ldots, \tilde{A}_{n}$ est "générique", si c'est un résultat d'une procédure suivante. On commence avec des algèbres disjointes $A_{1}, \ldots, A_{n}$ sur un corps $F$ telles que chaque $A_{i}$ satisfait la condition de 5.1. Puis, on prendre des $F$-algèbres $B_{1}, \ldots, B_{m}$ telles que leurs classes dans $\operatorname{Br}(F)$ appartiennent au sous-groupe engendré par $\left[A_{1}\right], \ldots,\left[A_{n}\right]$. Enfin, on prendre comme $Y$ un produit direct de variétés de Severi-Brauer généralisées quelconques des algèbres $B_{1}, \ldots, B_{m}$ et on pose $\tilde{A}_{i}=\left(A_{i}\right)_{F(Y)}$ pour tout $i=1, \ldots, n$.

Théorème 5.5. Si une collection d'algèbres $\tilde{A}_{1}, \ldots, \tilde{A}_{n}$ est "générique", le produit $\tilde{X}$ de leurs variétés de Severi-Brauer est une variété "générique" (4.1); en particulier, l'épimorphisme

$$
\text { Tors } \mathrm{G}^{2} \Gamma K(\tilde{X}) \rightarrow \text { Tors } \mathrm{CH}^{2}(\tilde{X})
$$

est bijectif dans ce cas.

Démonstration. Soient $A_{1}, \ldots, A_{n}$ les algèbres utilisées pour construction de notre collection "générique" (5.4). Posons $X_{i}=\operatorname{SB}\left(A_{i}\right)$ pour $i=1, \ldots, n$ et soit $X=X_{1} \times \cdots \times X_{n}$. D'après 5.2 , le groupe $\mathrm{G}^{*} \Gamma K(X)$ est sans torsion. En particulier, la variété $X$ est "générique" (4.2).

Maintenant, soit $Y$ le produit direct de variétés de Severi-Brauer généralisées, utilisé dans la construction de notre collection "générique". Par 5.3, la projection $X \times Y \rightarrow X$ est un produit fibré (sur $X$ ) de fibrations grassmanniennes. Donc, en utilisant (4.5) $m$ fois, on démontre que la variété $\tilde{X}=X_{F(Y)}$ est "générique".

Corollaire 5.6. Soient $A_{1}, \ldots, A_{n}$ des algèbres quelconques et $X$ le produit de leurs variétés de Severi-Brauer. Soit $\tilde{A}_{1}, \ldots, \tilde{A}_{n}$ une collection "générique" d'algèbres telles que $\operatorname{deg} \tilde{A}_{i}=\operatorname{deg} A_{i}$ et

pour tout $i$ et tous $j_{1}, \ldots, j_{n}$.

$$
\operatorname{ind}\left(\tilde{A}_{1}^{\otimes j_{1}} \otimes \cdots \otimes \tilde{A}_{n}^{\otimes j_{n}}\right)=\operatorname{ind}\left(A_{1}^{\otimes j_{1}} \otimes \cdots \otimes A_{n}^{\otimes j_{n}}\right)
$$

Le groupe Tors $\mathrm{CH}^{2}(X)$ est isomorphe à un groupe quotient de Tors $\mathrm{CH}^{2}(\tilde{X})$. 
Démonstration. Par le théorème, on a un isomorphisme

$$
\text { Tors } \mathrm{CH}^{2}(\tilde{X}) \simeq \text { Tors } \mathrm{G}^{2} \Gamma K(\tilde{X}) ;
$$

par 2.2 , on a

$$
\text { Tors } \mathrm{G}^{2} \Gamma K(\tilde{X}) \simeq \text { Tors } \mathrm{G}^{2} \Gamma K(X) ;
$$

enfin, on a toujours une surjection $([9,2.15])$

$$
\text { Tors } \mathrm{G}^{2} \Gamma K(X) \rightarrow \text { Tors } \mathrm{CH}^{2}(X)
$$

\section{VARIÉTÉ BIQUATERNIONIQUE FOIS CONIQUE}

Nous appelons variété biquaternionique une variété de Severi-Brauer d'une algèbre des biquaternions.

Théorème 6.1. Soient $X$ une variété biquaternionique, $Y$ une conique (sur le même corps) et $A, B$ les algèbres correspondantes ( $B$ est une algèbre des quaternions).

1. La torsion dans le groupe $\mathrm{CH}^{2}(X \times Y)$ est sois triviale, sois d'ordre 2 .

2. Si la torsion n'est pas triviale, alors

$$
\text { ind } A=\operatorname{ind}(A \otimes B)=4 \text { et } \quad \text { ind } B=2 \text {. }
$$

3. Si la collection $A, B$ est "générique" (5.4) et satisfait la condition (*), alors la torsion n'est pas triviale.

Démonstration. Si ind $B \neq 2$, c.-à.-d. si $B$ est décomposée, on sait par 1.1 que Tors $\mathrm{CH}^{2}(X \times Y) \simeq \mathrm{CH}^{2}(X)$; le dernier groupe n'a pas de torsion ([8, corollary]).

Si ind $A \neq 4$, alors $A$ est Brauer-équivalente à une algèbres des quaternions $A^{\prime}$; désignant par $X^{\prime}$ sa variété de Severi-Brauer, on obtient (1.1)

$$
\text { Tors } \mathrm{CH}^{2}(X \times Y) \simeq \text { Tors } \mathrm{CH}^{2}\left(X^{\prime} \times Y\right) \text {. }
$$

Puisque $\operatorname{dim}\left(X^{\prime} \times Y\right)=2$, le groupe

$$
\mathrm{G}^{2} \Gamma K\left(X^{\prime} \times Y\right)=\Gamma^{2} K\left(X^{\prime} \times Y\right) \subset K\left(X^{\prime} \times Y\right)
$$

n'a pas de torsion. On en conclut que Tors $\mathrm{CH}^{2}(X \times Y)=0$ aussi dans ce cas.

Soit $C$ l'algèbre à division qui est Brauer-équivalente au produit $A \otimes B$; $T=\mathrm{SB}(C)$. En utilisant 1.1 de nouveau, on tire

$$
\text { Tors } \mathrm{CH}^{2}(X \times Y) \simeq \text { Tors } \mathrm{CH}^{2}(T \times Y) \text {. }
$$

Si ind $(A \otimes B) \leq 2$, alors $\operatorname{dim} T \times Y \leq 2$ et on finit au même façon comme si-dessus.

Si ind $(A \otimes B)=8$, les algèbres $A, B$ sont disjointes et 5.2 montre que Tors $\mathrm{CH}^{2}(X \times Y)=0$.

Le reste est desservi par 
Proposition 6.2. Supposons que une algèbre des biquaternions $A$ et une algèbre des quaternions $B$ sont des algèbres à division et ind $(A \otimes B)=4$. Pour $X, Y$ comme ci-dessus, on a: Tors $\mathrm{G}^{2} \Gamma K(X \times Y) \simeq \mathbb{Z} / 2$.

Démonstration. Posons $K=K(X \times Y), \bar{K}=K(\bar{X} \times \bar{Y})$. L'anneau $\bar{K}$ est engendré par les elements $\xi, \eta$ avec les seules relations $(\xi-1)^{4}=0=(\eta-1)^{2}$ (v. §2). En particulier, le groupe additif de $\bar{K}$ est un groupe abélien librement engendré par les elements $\xi^{i} \eta^{j}, i=0,1,2,3, j=0,1$. Nous allons aussi utiliser un autre system de générateurs: $f^{i} g^{j}, i=0,1,2,3, j=0,1$, où $f=\xi-1$, $g=\eta-1$.

Pour chaque $l$, le l-ième term $\Gamma^{l} \bar{K}$ de la gamma-filtration de $\bar{K}$ est engendré par les produits $f^{i} g^{j}$ avec $i+j \geq l$. En particulier, $G^{l} \Gamma \bar{K}$ est un groupe abélien librement engendré par les classe résidueles des produits $f^{i} g^{j}$ avec $i+j=l$.

Lemme 6.3. Le sous-anneau $K \subset \bar{K}$ est addivement engendré par les elements

$$
1,4 \xi, \xi^{2}, 4 \xi^{3}, 2 \eta, 4 \xi \eta, 2 \xi^{2} \eta, 4 \xi^{3} \eta
$$

Démonstration. C'est 2.1 à notre situation particulier.

Lemme 6.4. Les elements suivants sont aussi des générateurs du groupe additif de $K$ :

$$
1,2 f-f^{2}, 2 g, 2 f^{2}, 4 f g, 4 f^{3}, 2 f^{2} g, 4 f^{3} g
$$

(l'element relevé va produir la torsion - v. 6.9).

Lemme 6.5. Il y a les inclusions suivantes:

$$
\begin{aligned}
& \Gamma^{1} K \ni 2 f-f^{2}, 2 g ; \\
& \Gamma^{2} K \ni 2 f^{2}, 4 f g, 2 f^{2} g ; \\
& \Gamma^{3} K \ni 4 f^{3}, 2 \cdot 2 f^{2} g ; \\
& \Gamma^{4} K \ni 4 f^{3} g .
\end{aligned}
$$

Démonstration. L'assertion sur $\Gamma^{1} K$ est evidente.

Puisque $2 f^{2}, 4 f g \in K \cap \Gamma^{2} \bar{K}$ et l'homomorphisme $\alpha^{1}$ est injectif ([16, lemme 6.3, (i)]), l'assertion sur $\Gamma^{2} K$ a lieu (une vérification direct (v. le rest de la démonstration) est aussi facile).

Finelment, on a:

$$
\begin{gathered}
c_{t}(4 \xi)=(1+f t)^{4} \quad \Rightarrow \quad c^{3}(4 \xi)=4 f^{3} \quad \Rightarrow \quad 4 f^{3} \in \Gamma^{3} K ; \\
2 f^{2} \in \Gamma^{2} K \text { et } 2 g \in \Gamma^{1} K \quad \Rightarrow \quad 4 f^{2} g=\left(2 f^{2}\right) \cdot(2 g) \in \Gamma^{3} K ; \\
c^{4}(4 \xi \eta)=(\xi \eta-1)^{4}=((f+1)(g+1)-1)=4 f^{3} g \in \Gamma^{4} K .
\end{gathered}
$$

Corollaire 6.6. Notons $\alpha^{*}$ l'homomorpshime de restriction $\mathrm{G}^{*} \Gamma K \rightarrow \mathrm{G}^{*} \Gamma \bar{K}$. Pour tout $i>0$, on $a: \operatorname{Im} \alpha^{i} \subset 2 \mathrm{G}^{i} \Gamma \bar{K}$. 
Démonstration. D'après le lemme, le groupe $\mathrm{G}^{\mathbf{1}} \Gamma K$ est engendré par les classes residueles des elements $2 f-f^{2}$ et $2 g$; leurs images dans $\mathrm{G}^{1} \Gamma \bar{K}$ sont effectivement divisibles par 2. Ainsi, l'assertion du corollaire pour $i=1$ est démontrée.

Puisque les elements de $\Gamma^{2} K, \Gamma^{3} K$ et $\Gamma^{4} K$ énumérés dans le lemme engendrent $\Gamma^{2} K$ et sont divisibles par 2 dans $\bar{K}$, on obtient l'assertion pour $i \geq 2$ (on utilise l'absense de torsion dans $\mathrm{G}^{*} \Gamma \bar{K}$ ).

Corollaire 6.7. \# Tors $\mathrm{G}^{*} \Gamma K \leq 2$.

Démonstration. Comme le groupe $\mathrm{G}^{*} \Gamma \bar{K}$ est sans torsion, Tors $\mathrm{G}^{*} \Gamma K \subset \operatorname{Ker} \alpha^{*}$. Nous allons montrer que \# $\operatorname{Ker} \alpha^{*} \leq 2$ en utilisant la formule suivante ([7, proposition]):

$$
\operatorname{Ker} \alpha^{*}=\# \operatorname{Coker} \alpha^{*} / \#(\bar{K} / K) \text {. }
$$

Il est facile de calculer que \# $(\bar{K} / K)=2^{10}$. D'après le lemme, \# Coker $\alpha^{*} \leq$ $2^{11}$.

Lemme 6.8. $2 f^{2} g \notin \Gamma^{3} K$.

Démonstration. Il suffit de montrer que $\operatorname{Im} \alpha^{3} \subset 4 \mathrm{G}^{3} \Gamma \bar{K}$.

Le groupe $\operatorname{Im} \alpha^{3}$ est engendré par le sous-groupe $\operatorname{Im} \alpha^{1} \cdot \operatorname{Im} \alpha^{2}$ est le sousensemble $\alpha^{3}\left(c^{3} K\right)$, où $c^{3}$ est la 3 -ième classe de Chern à valeurs dans $\mathrm{G}^{*} \Gamma K$ ([9, definition 2.7]). Puisque $\operatorname{Im} \alpha^{i} \subset 2 \mathrm{G}^{i} \Gamma \bar{K}$ pour $i>0$ par 6.6, on a: $\operatorname{Im} \alpha^{1}$. $\operatorname{Im} \alpha^{2} \subset 4 \mathrm{G}^{3} \Gamma \bar{K}$. Il suffit donc de vérifier que $\alpha^{3}\left(c^{3}(S)\right) \subset 4 \mathrm{G}^{3} \Gamma \bar{K}$ pour un system de generatuers du groupe additif de $K$. La vérification est triviale si on prend à titre de $S$ le system de generateurs de 6.3 .

Corollaire 6.9. Le résidu de $2 f^{2} g$ dans $\mathrm{G}^{2} \Gamma K$ a l'ordre 2 et engendre le sousgroupe de torsion.

Démonstration. Le résidu est d'ordre 2 d'après 6.8 et 6.5 . Il engendre tout le sous-groupe de torsion (non seulment de $\mathrm{G}^{3} \Gamma K$ mais aussi d'entier $G^{*} \Gamma K$ ) par 6.7 .

Nous avons fini les démonstrations du théorème et de la proposition.

Remarque 6.10. A la condition du théorème, notons $F$ le corps de base et supposons qu'il existe une extension quadratique $L / F$ (ou, plus généralement, une extension de degré indivisible par 4) telle que l'algèbre $A_{L}$ n'est plus une algèbre à division et l'algèbre $B_{L}$ est décomposée. Dans ce cas, $f^{2} g \in$ $\mathrm{T}^{3} K\left(X_{L} \times Y_{L}\right)$; en utilisant l'application de norme, nous obtenons: $2 f^{2} g \in$ $\mathrm{T}^{3} K(X \times Y)$, i.e. Tors $\mathrm{CH}^{2}(X \times Y)=0$.

Alors, si $A, B$ sont telles que Tors $\mathrm{CH}^{2}(X \times Y) \neq 0$ (par exemple, si $A, B$ forment une collection "générique" (6.1)), il n'y a pas d'extension comme sidessus. Le premier exemple semblable est construit dans [12]. 


\section{Produit de deuX surfaces de Severi-Brauer}

Un surface de Severi-Brauer est une variété de Severi-Brauer de dimension 2.

Théorème 7.1. Soient $X, Y$ des surfaces de Severi-Brauer sur un (même) corps et $A, B$ les algèbres correspondantes.

1. La torsion dans le groupe $\mathrm{CH}^{2}(X \times Y)$ est sois triviale, sois d'ordre 3 .

2. Si la torsion n'est pas triviale, alors

$$
\text { ind } A=\operatorname{ind} B=\operatorname{ind}(A \otimes B)=\operatorname{ind}\left(A \otimes B^{\circ}\right)=3
$$

où $B^{\circ}$ est l'algèbre opposée.

3. Si la collection $A, B$ est "générique" (5.4) et satisfait la condition (*), alors la torsion n'est pas triviale.

Démonstration. Si au moins une des algèbres $A, B, A \otimes B, A \otimes B^{\circ}$ est décomposée, il existe une algèbre $C$ du degré 3 telle que sa classe $[C]$ dans le groupe de Brauer engendre le même groupe que $[A]$ et $[B]$ (ensemble). D'après 1.1 , le groupe Tors $\mathrm{CH}^{2}(X \times Y)$ est isomorphe dans ce cas au groupe Tors $\mathrm{CH}^{2}(\mathrm{SB}(C))$ qui est trivial par [7, corollary].

Si ind $(A \otimes B)=\operatorname{ind}\left(A \otimes B^{\circ}\right)=9$, alors les algèbres $A, B$ sont disjointes et on peut utiliser 5.2 .

Posons $Y^{\mathrm{o}}=\mathrm{SB}\left(B^{\mathrm{o}}\right)$. Comme par (1.1)

$$
\text { Tors } \mathrm{CH}^{2}(X \times Y) \simeq \text { Tors } \mathrm{CH}^{2}\left(X \times Y^{\circ}\right),
$$

il suffit de regarder seulment un des deux cas suivants:

- $\operatorname{ind}(A \otimes B)=3$ et $\operatorname{ind}\left(A \otimes B^{\circ}\right)=9$;

- $\operatorname{ind}(A \otimes B)=9$ et $\operatorname{ind}\left(A \otimes B^{\circ}\right)=3$.

Lemme 7.2. $S i$ ind $A=\operatorname{ind} B=\operatorname{ind}(A \otimes B)=3$ et $\operatorname{ind}\left(A \otimes B^{\circ}\right)=9$, alors Tors $\mathrm{G}^{2} \Gamma K(X \times Y)=0$.

Démonstration. Posons $K=K(X \times Y), \bar{K}=K(\bar{X} \times \bar{Y})$. L'anneau $\bar{K}$ est engendré par les elements $\xi, \eta$ avec les relations $(\xi-1)^{3}=0=(\eta-1)^{3}$ (v. $\S 2)$. En particulier, le groupe additif de $\bar{K}$ est un groupe abélien librement engendré par les elements $\xi^{i} \eta^{j}, i, j=0,1,2$. Nous allons aussi utiliser un autre system de générateurs: $f^{i} g^{j}, i, j=0,1,2$, où $f=\xi-1, g=\eta-1$.

Pour chaque $l$, le $l$-ième term $\Gamma^{\prime} \bar{K}$ de la gamma-filtration de $\bar{K}$ est engendré par les produits $f^{i} g^{j}$ avec $i+j \geq l$.

La condition du lemme implique que

$$
\begin{gathered}
\text { ind } A^{\otimes 2}=\operatorname{ind} B^{\otimes 2}=\operatorname{ind}\left(A^{\otimes 2} \otimes B^{\otimes 2}\right)=3 \text { et } \\
\operatorname{ind}\left(A \otimes B^{\otimes 2}\right)=\operatorname{ind}\left(A^{\otimes 2} \otimes B\right)=9 .
\end{gathered}
$$

Ainsi, d'après 2.1 , le sous-anneau $K \subset \bar{K}$ est additivement engendré par

$$
1,3 \xi, 3 \xi^{2}, 3 \eta, 3 \xi \eta, 9 \xi^{2} \eta, 3 \eta^{2}, 9 \xi \eta^{2}, 3 \xi^{2} \eta^{2}
$$


Nous allons aussi utiliser un autre system de générateurs:

$$
1,3 f, 3 g, 3 f^{2}, 3 f g, 3 g^{2}, 9 f^{2} g, 3 f^{2} g+3 f g^{2}+6 f^{2} g^{2}, 9 f^{2} g^{2} \text {. }
$$

Maintenant c'est evident que l'intersection $K \cap \Gamma^{3} \bar{K}$ est engendrée par

$$
9 f^{2} g, 3 f^{2} g+3 f g^{2}+6 f^{2} g^{2} \text { et } 9 f^{2} g^{2} \text {. }
$$

Pour démontrer que le groupe $\mathrm{G}^{2} \Gamma K$ est sans torsion, il suffit de vérifier que les trois elements appartiennent à $\Gamma^{3} K$.

Comme $3 f^{2}, 3 g^{2} \in \Gamma^{2} K$ et $3 g \in \Gamma^{1} K$, on a:

$$
9 f^{2} g=\left(3 f^{2}\right) \cdot(3 g) \in \Gamma^{3} K, 9 f^{2} g^{2}=\left(3 f^{2}\right) \cdot\left(3 g^{2}\right) \in \Gamma^{4} K .
$$

L'element resté coïncide avec une 3-ième classe de Chern:

$$
\begin{aligned}
c^{3}(3 \xi \eta)=(\xi \eta-1)^{3}=((f+1)(g+1)-1)^{3}=(f g+f+g)^{3}= \\
3 f g(f+g)^{2}+(f+g)^{3}=6 f^{2} g^{2}+3 f^{2} g+3 f g^{2}
\end{aligned}
$$

Nous finissons la démostration du théorème avec

Proposition 7.3. $S i$ ind $A=\operatorname{ind} B=\operatorname{ind}(A \otimes B)=\operatorname{ind}\left(A \otimes B^{\circ}\right)=3$, alors Tors $\mathrm{G}^{2} \Gamma K(X \times Y) \simeq \mathbb{Z} / 3$.

Démonstration. Nous utilisons la notation introduite au commencement de la démonstration du dernier lemme.

Lemme 7.4. Le sous-anneau $K \subset \bar{K}$ est maintenant engendré par 1 et $3 \bar{K}$. En plus,

$$
\begin{aligned}
& \Gamma^{1} K=3 \Gamma^{1} \bar{K} \\
& \Gamma^{2} K=3 \Gamma^{2} \bar{K} \\
& \Gamma^{3} K \ni 3 f^{2} g-3 f g^{2}, 3 f^{2} g+3 f g^{2}+6 f^{2} g^{2} \\
& \Gamma^{4} K \ni 9 f^{2} g^{2}
\end{aligned}
$$

Démonstration. L'assertion sur $\Gamma^{1} K$ est triviale. L'assertion sur $\Gamma^{2} K$ découle d'injectivité de $\alpha^{1}$ ([16, lemme 6.3, (i)]); puis $9 f^{2} g^{2} \in \Gamma^{4} K$ car $3 f^{2}, 3 g^{2} \in \Gamma^{2} K$.

Pour montrer l'assertion sur $\Gamma^{3} K$, calculons la 3 -ième classe de Chern

$$
c^{3}\left(\xi^{2} \eta\right)=\left(\xi^{2} \eta-1\right)^{3}=\left((f+1)^{2}(g+1)-1\right)^{3}=27 f^{2} g^{2}+12 f^{2} g+6 f g^{2} \text {. }
$$

Puisque $9 f^{2} g, 9 f g^{2} \in \Gamma^{3} K$, on en conclude que $3 f^{2} g-3 f g^{2} \in \Gamma^{3} K$.

Enfin, comme nous avons deja calculé dans le preuve de 7.2,

$$
3 f^{2} g+3 f g^{2}+6 f^{2} g^{2}=c^{3}(3 \xi \eta) \in \Gamma^{3} K
$$

Corollaire 7.5. \# Tors $\mathrm{G}^{*} \Gamma K \leq 3$.

Démonstration. Analogiquement 6.7. Maintenant, on a: $\#(\bar{K} / K)=3^{8}$ et \# Coker $\alpha^{*} \leq 3^{9}(7.4)$. 
Lemme 7.6. $3 f^{2} g^{2} \notin \Gamma^{3} K$.

Démonstration. Définissons un homomorphisme $\phi_{9}: \bar{K} \rightarrow \mathbb{Z} / 9$ à la maniere suivante: ecrivons un élément $x \in \bar{K}$ arbitraire comme une combination lineaire

$$
x=\sum_{i, j=0}^{2} a_{i j} f^{i} g^{j} \quad \text { avec } a_{i j} \in \mathbb{Z},
$$

posons $\phi(x)=a_{21}+a_{12}+a_{22}$ et définissons $\phi_{9}(x)$ comme le résidu de $\phi(x)$ modulo 9 .

Comme $\phi_{9}\left(3 f^{2} g^{2}\right) \neq 0$, il suffit de montrer que $\phi_{9}\left(\Gamma^{3} K\right)=0$.

A priori, le groupe $\Gamma^{3} K$ est engendré par $\Gamma^{1} K \cdot \Gamma^{2} K, c^{3}(S)$ et $c^{4}(S)$ où

$$
S=1,3 \xi, 3 \xi^{2}, 3 \eta, 3 \xi \eta, 3 \xi^{2} \eta, 3 \eta^{2}, 3 \xi \eta^{2}, 3 \xi^{2} \eta^{2} .
$$

Cependant, $c^{4}(s)=0$ pour tout $s \in S$; on peut donc rayer $c^{4}(S)$ de la liste des generateurs.

Puisque

$$
\Gamma^{1} K \cdot \Gamma^{2} K \subset \Gamma^{1} K \cdot \Gamma^{1} K \subset 9 \bar{K} \quad(7.4),
$$

on a: $\phi_{9}\left(\Gamma^{1} K \cdot \Gamma^{2} K\right)=0$.

Il reste $c^{3}(S)$. Pour $s=1,3 \xi, 3 \xi^{2}, 3 \eta$ et $3 \eta^{2}, \phi(s)$ est deja 0 . Les calculs suivants montrent que $\phi_{9}\left(c^{3}(s)\right)=0$ aussi pour les autres quatre elements $s \in S$ :

$$
\begin{aligned}
& c^{3}(3 \xi \eta)=(\xi \eta-1)^{3}=((f+1)(g+1)-1)=(f g+(f+g))^{3}= \\
& =3 f g(f+g)^{2}+(f+g)^{3}=6 f^{2} g^{2}+3 f^{2} g+3 f g^{2} \text {; } \\
& c^{3}\left(3 \xi^{2} \eta^{2}\right)=\left(\xi^{2} \eta^{2}-1\right)^{3}=\left((f+1)^{2}(g+1)^{2}-1\right)=\left(\left(f^{2}+4 f g+g^{2}\right)+2(f+g)\right)^{3}= \\
& =12\left(f^{2}+4 f g+g^{2}\right)(f+g)^{2}+8(f+g)^{3}=120 f^{2} g^{2}+24 f^{2} g+24 f g^{2} \text {; } \\
& c^{3}\left(3 \xi^{2} \eta\right)=\left(\xi^{2} \eta-1\right)^{3}=\left((f+1)^{2}(g+1)-1\right)^{3}=(f(f+2 g)+(2 f+g))^{3}= \\
& =3 f(f+2 g)(2 f+g)^{2}+(2 f+g)^{3}=27 f^{2} g^{2}+12 f^{2} g+6 f g^{2} ; \\
& c^{3}\left(3 \xi \eta^{2}\right) \\
& =27 f^{2} g^{2}+6 f^{2} g+12 f g^{2}
\end{aligned}
$$




\section{RÉFÉRENCES}

[1] Blanchet, A. Function fields of generalized Brauer-Severi varieties. Comm. Algebra 19.1 (1991), 97-118.

[2] Fulton, W. Intersection Theory. Springer-Verlag, 1984.

[3] Fulton, W., Lang, S. Riemann-Roch Algebra. Springer-Verlag, 1985.

[4] Hartshorne, R. Algebraic Geometry. Springer-Verlag, 1977.

[5] Izhboldin, O. T. On the (non)-excellent property for function field of Severi-Brauer varieties. Preprint.

[6] Karpenko, N. A. Algebro-geometric invariants of quadratic forms. Leningrad (St. Petersburg) Math. J. 2 (1991), no. 1, 119-138.

[7] Karpenko, N. A. On topological filtration for Severi-Brauer varieties. Proc. Symp. Pure Math. 58.2 (1995), 275-277.

[8] Karpenko, N. A. On topological filtration for Severi-Brauer varieties II. Amer. Math. Soc. Transl. (2) Vol. 174, 1996, 45-48.

[9] Karpenko, N. A. Codimension 2 cycles on Severi-Brauer varieties. Prépublications de l'Équipe de Mathémathiques de Besançon 96/40 (1996), 26 p.

[10] Karpenko, N. A., Merkurjev, A. S. Chow groups of projective quadrics. Leningrad (St. Petersburg) Math. J. 2 (1991), no. 3, 655-671.

[11] Köck, B. Chow motif and higher Chow theory of G/P. Manuscripta Math. 70 (1991), 363-372.

[12] Mammone, P. On the tensor product of division algebras. Arch. Math. 58 (1992), 34-39.

[13] Manin, Yu. I. Lectures on the K-functor in algebraic geometry. Russian Math. Surveys 24 (1969), no. 5, 1-89.

[14] Peyre, E. Products of Severi-Brauer varieties and Galois cohomology. Proc. Symp. Pure Math. 58.2 (1995), 369-401.

[15] Quillen, D. Higher algebraic K-theory: I. Lect. Notes Math. 341 (1973), 85-147.

[16] Sansuc, J.-J. Groupe de Brauer et arithmétique des groupes algébriques linéaires sur un corps de nombres. J. reine angew. Math. 327 (1981), 12-80.

Université de Franche-Comté, Équipe de Mathématiques de Besançon, 16, RouTe DE GraY, F-25030 BESANÇON CEDEX, FRANCE

E-mail address: karpenkodmath.univ-fcomte. Ir 